\title{
Determination of serological profiles and avidity of specific antibodies in the sera of patients with potential Epstein-Barr virus (EBV) infection
}

\author{
Akpolat $\mathrm{N}^{1}$, Gedik $\mathrm{M}^{2}$, Nergiz $\mathrm{S}^{1}$, Bilek $\mathrm{H}^{3}$ \\ Department of Microbiology, Faculty of Medicine, Dicle University, Diyarbakir, Turkey. sebnemnergiz@gmail.com
}

\begin{abstract}
Background: Epstein-Barr Virus, frequently referred to as EBV, is a member of the herpesvirus family and one of the most common human viruses. The virus occurs worldwide, and most people become infected with EBV sometime during their lives.

Objectives: The purpose of this study was to investigate the serological profiles of specific antibodies among the sera of suspected EBV infection patients along with VCA-IgG avidity.

Methods: A total of 522 patient's sera were sent to The Clinical Microbiology Laboratory for EBV specific antibody detection and were studied by IFA method during a two year period. The serum samples were tested for EBV specific VCA IgG, VCA IgM, EA, EBNA antibodies and VCA IgG aviditity.

Results: Among 33 patients those who had low avidity for VCA IgG, $27(81.8 \%)$ of them had a serologic profile as follows; positive VCA IgG, negative VCA IgM, negative EA and negative EBNA.

Conclusion: While this profile is considered as a primary infection, the frequency of the coexistence of VCA IgG low avidity with this profile is interepreted that avidity may lead to detect primary infection (Tab. 2, Ref. 25). Full Text in PDF www.elis.sk.

Key words: EBV infection, VCA IgG avidity.
\end{abstract}

Abbreviations: IFA - indirect fluorescent antibody, EBV - Epstein-Barr virus, EA - early antigen, VCA - viral capsid antigen, EBNA - epstein-barr nuclear antigen.

Epstein-Barr virus, commonly referred to as EBV, is a human specific member of the herpesvirus family and is a B-cell lymphotropic virus. An EBV infection occurs by close contact with EBV presented in oral secretions. The virus initially wells in the oropharyngeal epithelial cells and causes persistent infections. Seroepidemiologic studies indicate that $\mathrm{EBV}$ infections are common in all communities and occur worldwide, and most people become infected with EBV sometime during their lives. In Europe including Turkey, as many as 70-80\% of adolescents and $80-90 \%$ of adults are reported to be seropositive for EBV (1).

The course of EBV infection can vary with regard to the age and health status of the person. In young children host-virus infection can take place with no apparent symptoms. By contrast, in $30-50 \%$ of the adult patients EBV infection can cause diffuse

${ }^{1}$ Department of Microbiology, Faculty of Medicine, Dicle University, Diyarbakir, Turkey, ${ }^{2}$ Department of Microbiology, Yalova State Hospital, Yalova, Turkey, and ${ }^{3}$ Department of Microbiology, Sirnak State Hospital, Sirnak, Turkey

Address for correspondence: Nergiz S, MD, Department of Microbiology, Faculty of Medicine, Dicle University, Diyarbakir 21280, Turkey.

Phone: +90.412 .2488001$ lymphadenopathy, hepatomegaly, splenomegaly, and tonsillitis. In some cases exanthematous reactions triggered by EBV can be mistaken with an eruption of rubella (2). Moreover, EBV infection is shown to be associated with lymphoproliferative disorders in patients who are suffering from immunodeficiency, Burkitt lymphoma, and nasopharyngeal carcinoma $(3,4)$. Infectious mononucleosis is also caused by infection of B cells by EBV and is generally diagnosed by serological methods. EBV serology is utilized to help distinguish EBV reactivation infections from primary EBV infections and to demonstrate acute EBV infections. During this process, it is critically important to perform the tests at the same time for the presence of EBV-VCA IgM, EBV-VCAIgG, anti-EBV-EA, anti EBV-EBNA and VCA IgG avidity (2).

The spectrum of antibody assays comprises unspecific tests, such as the long-known test for the detection of heterophile antibodies, as well as EBV-specific assays. Commercially available agglutination test kits for the detection of heterophile antibodies use goat, horse, or bull red blood cells after preabsorption with guinea pig kidney extracts and are effective with acute-phase serum for 85 to $90 \%$ of adolescent or adult patients but are only 50 $\%$ effective for children ages 2 to 5 years. Thus, fairly high rates of false-negative results may be expected, while false-positive results were found in 2 to $3 \%$ of patients with autoimmune diseases (5). Today, EBV-specific serology is preferred for the diagnosis of an EBV infection, but in the absence of heterophile antibodies. Unfortunately, EBV-specific diagnostic tests are not standardized. 
The EBV-specific assays differ in the substrates or antigens and the technologies that they use. So far, three methods serve as the method of first choice in routine EBV diagnostics: the IFA, which is still the gold standard method; different enzyme immunoassay (EIA) techniques, including solid-phase ELISAs and related methods, such as luminescence-based detection of anti-EBV antibodies with antigen-coated beads; and Western blot analysis. While IFA or EIA is often used for screening, Western blot analysis is mainly performed for confirmation (6). Here we investigate the serological profiles of specific antibodies among the sera of suspected EBV infection patients along with VCA-IgG avidity by IFA method.

\section{Materials and methods}

The study was performed during a two-year period in the Department of Microbiology at the Medical School of Dicle University, Diyarbakir, Turkey. We received the sera of 522 patients who were suspected to have an EBV infection for the detection of EBV specific antibodies. The sera were tested for the presence of VCAIgG, VCA-IgM, EA and EBNA specific antibodies, and VCA-IgG avidity. The specific antibodies were determined via an Indirect Fluorescent Antibody (IFA) technique using the commercial kit (EUROIMMUN BIOCHIP Sequence EBV Avidity Determinant; Lot: F080514VC, Germany). This kit can both, qualitatively and quantitatively determine antibodies and evaluate the avidity of specific antibodies in serum and plasma. The working slides for the test are established using the human EBV transformed cell lines obtained from Burkitt lymphoma patients, and the cell lines P3HR1 expressing VCA, Raji cell expressing EBNA complex, and EU-33 expressing EA antigen.

For the assay briefly, patient samples were diluted 1:10 in PBS-Tween. $30 \mu 1$ of diluted serum was applied to each reaction area of the reagent tray. Then, the slides were placed into the corresponding recesses of the reagent tray. The BIOCHIPs came into contact with the droplets and the reaction was started. The slides were incubated for 60 minutes at room temperature. Following the incubation, the slides were reacted with FITC-labeled anti-human IgG/IgM (goat). Results were evaluated with a fluorescence microscope (Zeiss, Germany) by using a 20x objective.

Moreover, we determined anti-EBNA antibodies using an anti-complement indirect immunofluorescence technique. Antibodies of class IgG should be removed from the patient samples by immunoabsorption (EUROSORB; EUROIMMUN, Germany) before determining specific antibodies of class IgM. This prevents non-specific IgM binding.
In order to identify low- avidity antibodies in patient serum, two indirect immunofluorescence tests are performed in parallel: one test is performed in the usual way, and in the other a urea ( 8 $\mathrm{M})$ treatment is carried out between the incubations with patient serum and FITC-labelled anti-human IgG. This releases the lowavidity antibodies from antigen, while high-avidity antibodies remain bound. To determine the antibody avidity the fluorescence intensity of the incubated patient samples without urea treatment is compared with the fluorescence intensity of the same serum sample treated with urea. The intensity of the specific fluorescence is differentiated by the scoring scheme: 0 no fluorescence, 1 weak fluorescence intensity, 2 moderate fluorescence intensity, 3 strong fluorescence intensity and 4 very strong fluorescence intensity.

If the specific fluorescence of the incubated serum sample without urea treatment is assessed as 0,1 or 2 , it is not possible to determine the avidity determination. If the difference between the fluorescence intensity scores is less than two, there are generally high- avidity antibodies present. If the difference between the fluorescence intensity scores is two or more, there are generally low- avidity antibodies present.

\section{Results}

We studied EBV specific antibodies in the sera of 522 patients using the IFA technique at our hospital during a two-year period. Overall, 200 (38.3\%) of the patients were female and $322(61.7 \%)$ of them were male. Of all analyzed sera, we found the samples of 94 patients (18.1\%) negative for all EBV specific antibodies and these patients were accepted as seronegative. The rest of the sera $(82 \%, n=428)$ were found to be seropositive for EBV specific antibodies. Of 428 seropositive patients, 170 (39.7\%) were female and $258(60.3 \%)$ were male. We observed the highest rate for seropositivity ( $40.88 \%$ ) among patients in 6-12 age group. In this age group, the seropositivity ratio was $37.6 \%$ for female and $43.02 \%$ for male patients.

In addition, 265 (61.9\%) seropositive patients had high IgG avidity, while $33(7.7 \%)$ had low IgG avidity. The distribution of age and sex among low avidity VCA-IgG detected patients is summarized in Table 1. All of these patients were seronegative for EBNA antibodies. Twenty seven $(81.8 \%$ ) patients were found to be positive for VCA-IgG but negative for VCA-IgM, EA, and EBNA (Group A). Five patients showed positive EA but negative VCA-IgM and EBNA serological profiles (Group B). One patient possessed positive VCA-IgM and EA but negative EBNA profil (Group C). The results of the test of Anti-EBV antibodies are summarized in Table 2.

Tab. 1. The distribution of age and sex among low avidity VCA-IgG detected patients.

\begin{tabular}{|c|c|c|c|c|c|c|}
\hline \multirow[t]{2}{*}{ Age Groups } & \multicolumn{2}{|c|}{ Female } & \multicolumn{2}{|c|}{ Male } & \multicolumn{2}{|c|}{ Total } \\
\hline & Number & $\%$ & Number & $\%$ & Number & $\%$ \\
\hline$\overline{0-2}$ & 1 & 3.03 & 2 & 6.06 & 3 & 9.09 \\
\hline $3-5$ & 6 & 18.3 & 5 & 15.2 & 11 & 33.3 \\
\hline $6-12$ & 1 & 3.03 & 12 & 36.4 & 13 & 39.4 \\
\hline $13-17$ & 1 & 3.03 & 2 & 6.06 & 3 & 9.09 \\
\hline$\geq 17$ & 0 & 0 & 3 & 9.09 & 3 & 9.09 \\
\hline Total & 9 & 27.3 & 24 & 72. & 33 & 100 \\
\hline
\end{tabular}


$460-463$

Tab. 2. The results of the test of Anti-EBV antibodies.

\begin{tabular}{|c|c|c|c|c|c|}
\hline No.of samples & VCA IgG/avidity & VCA IGM & EBNA & EA & Group \\
\hline $27 / 428$ & positive/low & negative & negative & negative & $\mathrm{A}$ \\
\hline $5 / 428$ & positive/low & negative & negative & positive & B \\
\hline $1 / 428$ & positive/low & positive & negative & positive & $\mathrm{C}$ \\
\hline $33 / 428$ & positive/low & & negative & & \\
\hline
\end{tabular}

Studying the maturation of the antibody for the patients would be meaningful but we failed to pursue this. Furthermore, thirty three patients were interpreted to have a primary infection. Nine patients were referred to the laboratory for upper respiratory airway infections and 18 patients were sent to the laboratory for lymphadenopathy indication but we failed to reach the medical records of 6 patients. The distribution of patients identified with low VCA-IgG avidity with regard to their age and gender is illustrated in Table 1.

\section{Discussion}

Although the presence of VCA-IgM in serum indicates a primary infection, studies show that discrete serological responses can exist in EBV infections $(6,7)$. The production of IgM in EBV infections may not be present in some adult patients or may be generated later. Moreover, EBV can become a persistent infection after a long time or can be reactivated in immunosuppressive patients $(7,8)$. Therefore, it is difficult to decide whether an infection is a secondary infection through the finding of specific IgM positivity in serum samples and if this infection is due to persistent IgM or reactivation/re-infection. In addition, the presence of IgM negativity resulting from inappropriate timing of blood sampling or the loss of EBNA antibodies owing to a suppressed immune system can obscure the identification of primary infections or can lead to misdiagnosis. In such cases, it is suggested that the study of IgG avidity is critical to interpreting serological results correctly (9-12). In general, the kinetics of affinity maturation occurs in a couple of weeks; however, in some cases this maturation process may arise within three months after the onset of symptoms. This alteration in avidity is also described in several infections such as Rubella, Toxoplasmosis, and hepatitis B $(5,13)$. Avidity is established through the affinity maturation of antibodies to immune response and it is thought that higher avidity goes together with the potent functional activities of antibodies $(14,15)$. The increase in antibody avidity stabilizes immune complexes (antigen-antibody complexes) against protein denaturing agents present in the environment and these agents can dissociate low affinity antibodies from immune complexes readily. Based on this basic knowledge we can calculate antibody affinity using protein denaturing agents such as urea, diethylamine, guanine hydrochloride and potassium thiocyanate in immunological tests (16-20). Among these, the simplest method is the use of $8 \mathrm{M}$ urea. Moreover, the determination of specific $\mathrm{IgG}$ antibody avidity is performed thorough various techniques including agglutination, radioimmunoassay, complement fixation, ELISA, immunofluorescence, electroblotting, immunoblot (16). However, some studies suggest that immunoblot is easier to interpret, as compared to ELISA and is the preferred method for measuring avidity $(12,21)$. Immunofluorescence assay (IFA) is used widely for detecting EBV-specific antibodies because of its high sensitivity and specificity. Consequently, IFA was accepted as the reference assay for detecting EBV antibodies (6). The EIA was less sensitive than the IFA for IgG detection early in the course of acute EBV infections. In a previous comparison of EBV antibody avidity tests based on a VCA IFA and on an antigen mixture EIA both methods gave good results, but EIA avidity was easier to carry out and showed a better distinction between acute and past EBV infections (22). However, the EIA was less sensitive than the IFA for IgG detection early in the course of acute EBV infections. Denaturing agents other than urea may facilitate a full automation of avidity testing in the future (6).

Our findings show that $33(7.71 \%)$ of 428 seropositive patients not only had low avidity IgG but also were seronegative for EBNA antibodies. Of these 33 patients, 27 (81.8\%) were found to be positive for VCA-IgG but negative for VCA-IgM, EA and EBNA. These results were inferred as primary infections and the accompanying low avidity VCA-IgG to this profile further indicates the presence of a primary infection. For the rest of the 33 patients with low avidity $\mathrm{IgG}$, the discovery of positive EA but negative VCA-IgM and EBNA serological profile of five patients, and the presence of positive VCA-IgM and EA but negative EBNA profile for one patient also support the existence of primary infections in these patients.

Grey showed, for the first time in 1996 that changes in avidity happen against VCA-EBV (5). Low avidity antibodies are shown to be present in $90 \%$ of primary infections within a 10 day period after the appearance of symptoms. Low avidity antibodies can also be seen in $50 \%$ of patients 30 days after the beginning of the primary infection. Moreover, Anderson et al (23) investigated the maturation kinetics of VCA-IgG avidity using the IFA technique in 105 patients with primary EBV infections. Their study showed that over $90 \%$ percent of the patients possessed a low avidity index (0.25) during the first 10 days of the infection and $50 \%$ of the patients still showed a low avidity index 20-30 days after the manifestation of clinical symptoms. They also observed that the avidity index increased (0.5-1) in the persons who were passed the infection. Therefore, this study emphasizes that antibody avidity is a critical serological determinant in EBV infections to determine whether they are acute or passed infections.

Similar to other studies, Robertson et al (5) emphasizes the importance of antibody avidity in the diagnosis of primary EBV infections as well. They studied VCA-IgG avidity using ELISA in the serums of 28 patients with primary EBV infections and in 35 patients with passed EBV infections. Their study demonstrates that the sensitivity of antibody avidity in the diagnosis of primary EBV infection is between $93-100 \%$ (100\%: particularly in the absence of EBNA but in the presence of $\operatorname{IgG})$. 
Furthermore, according to their study after finding IgM class antibodies against EBV, Rubella, CMV, and HSV in the first blood sample taken from a patient with infectious mononucleosis, Topkaya et al (24) investigated the presence of IgG subclass antibodies developed against these viruses and found that low avidity VCA-IgG was present only in EBV infection. After including other serological tests related to EBV, they diagnosed the patient with infectious mononucleosis. At their one month follow-up, they reported that IgM class antibodies were negative in Rubella, CMV, and HSV infections but EBV-VCA IgG avidity was increased. The authors conclude that it is critical to study not only IgM, IgG class antibodies but antibody avidity tests for the accurate differential diagnosis in the patients suspected to suffer from exanthema and infectious mononucleosis.

It should be useful to compare our results with a line immunoassay (recomLine EBV IgG), which is recommended recently for detection of IgG-antibodies. The test is based on nitrocellulose strips coated with recombinant proteins p54 and p138 (EA), p23 and p18 (VCA) and p72 (EBNA-1) (25).

In conclusion, the present study further supports earlier studies and suggests that a thorough assessment of specific IgG avidity is notable for the accurate inference of serological profiles of EBV infection and the precise diagnosis of infectious mononucleosis. Further studies are needed on this issue. It is important that our work creates an infrastructure for further studies.

\section{References}

1. Arman D. Epstein-Barr virus. 1197-201. In: Wilke Topçu A, Söyletir G, Doğanay M (Eds). Infectious Diseases and Microbiology. İstanbul; Nobel Press, 2002.

2. Shooley RT. Epstein-Barr virus infections (Infectious Mononucleosis). 1599-613. In: Mandell GL, Bennett Se, Dolin R (Eds). Principles and Practice of infectious Diseases. 4 ed. New York; Churchill Livingstone, 2000.

3. Klutts JS, Liao RS, Dunne Wm, Gronowski AM. Evaluation of a multiplex bead assay for assesment of Epstein-Barr Virus immunologic status. J Clin Microbiol 2004; 42: 4996-5000.

4. Klein E. Non-proliferative interactions of Epstein-Barr Virus and Human B Lymphocytes. Folia Biologica (Praha) 2004; 50: 131-135.

5. Robertson P, Beynon S, Whybin R, Brennan C, Vollmer CV, Hickie I, Lloyd A. Measurement of EBV-IgG anti VCA avidity aids the early and reliable diagnosis of primary EBV infection. J Med Virol 2003; 70 (4): 617-623.

6. Hess RD. Routine Epstein-Barr Virus diagnostics from the laboratory perspective: still challenging after 35 Years. J Clin Microbiol 2004; 42 (8): $3381-3387$.

7. Paul JR, Bunnell WW. The presence of heterophile antibodies in infectious mononucleosis. Am J Med Sci 1974; 267: 178-88.

8. Jenum PA, Pedersen BS, Gundersen AG. Improved diagnosis of primary Toxoplasma gondii infection in early pregnancy by determination of antitoxoplasma immunoglobulin G Avidity J Clin Microbiol 1997; 35: 1972-1977.
9. Horwitz CA, Henle W, Henle G, Schapiro R, Borken S, Bundtzen R. Infectious mononucleosis in patients aged 40-72 years: report of 27 cases, including 3 without heterophile antibody responses. Medicine 1983; 62: 256-262.

10. Naveau S, Delfraissy JF, Poitrine A, Poynard T, Chaput JC. Simultaneous detection of IgM antibodies against the hepatitis A virus and the viral capsid antigen of Epstein Barr virus in acute hepatitis. Gastroenterol Clin Biol 1985; 9: 109-112.

11. Sensini A, Pasceli S, Marchetti D, Castronori R, Marangi M, Sbaraglia M, Mottola A. IgG avidity in the serodiagnosis of acute Toxoplasma gondii infection; a multicenter study. Clin Microbiol and Infec 1996; 2: 25-29.

12. Weissbrich B. The use of semi-automated EBV IgG avidity determination for the diagnosis of infectious mononucleosis. J Med Virol 1998; 54:145-53.

13. Lappalainen M, Koskela P, Keskiniemi M. Toxplasmosis acquired during pregnancy; improved serodiagnosis based on avidity of IgG. Infect. Dis 1993; 167: 691-697.

14. Rauer S, Beitlich P, Neubert U, Rasiah C, Kaiser R. Avidity determination of Borrelia burgdorferi specific IgG antibodies in Lyme disease. Scand J Infect Dis 2001; 33 : 809-811.

15. Wuorimaa T, Dagan R, Vakevainen $M$, et al. Avidity and subclasses of IgG after immunization of infants with an 11-valent pneumococcal conjugate vaccine with or without aluminium adjuvant. J Infect Dis 2001; 184: $1211-1215$.

16. Gutierrez J, Maroto C. Are IgG antibody avidity assays useful in the diagnosis of infectious diseases. Microbios 1996; 87: 113-121.

17. Çolak D. Immunoglobulin G avidity tests in the diagnosis of infectious diseases. ANKEM Derg 2001; 15: 621-624.

18. Korhonen MH, Brunstein J, Haario H, Katnikov A, Rescaldani R, Hedman K. A new method with general diagnostic utility for the calculation of immunoglobulin G avidity. Clin Diag Lab Immunol 1999; 6: $725-728$.

19. Jawetz E, Melnick JL, Adelberg EA(Eds). Review of Medical Microbiology. 7th ed. Connecticut: Lange Medical Publications, 1987; 170-190.

20. Prince HE, Wilson M. Simplified assays for measuring Toxoplasma gondii IgG avidity. Clin Diag Lab Immunol 2001; 8: 904-908.

21. Strasek K, Marin J. Epstein-Barr virus infections avidity test for IgG antibodies. Zdrav Vestn 2001; 70: 321-323.

22. Schubert $\mathbf{J}$, Zens $\mathbf{W}$ and Weissbrich B. Comparative evaluation of the use of immunoblots and of $\operatorname{IgG}$ avidity assays as confirmatory tests for the diagnosis of acute EBV infections. J Clin Virol 1998; 11: 161-172.

23. Anderson A, Vetter V, Kreutzer L, Bauer G. Avidities of IgG directed against viral capsid antigen or early antigen: useful markers for significant Epstein-Barr virus serology. J Med Virol 1994; 43: 238-244.

24. Topkaya AE, Aksungar FB, Özakkaş F, Akıncı NÇ. An infectious mononucleosis case. J Med Sci 2007; 27 (2): 279-81.

25. Nystad TW, Myrmel H. Prevalence of primary versus reactivated Epstein-Barr virus infection in patients with VCA IgG, VCA IgM and EBNA-1 antibodies and suspected infectious mononucleosis. J Clin Virol 2007; 38: 292-297. 